\title{
A generalisation of Mahler measure and its application in algebraic dynamical systems
}

by

\author{
MANFRED EINSIEDLER (Wien)
}

We prove a generalisation of the entropy formula for certain algebraic $\mathbb{Z}^{d}$-actions given in [2] and [4]. This formula expresses the entropy as the logarithm of the Mahler measure of a Laurent polynomial in $d$ variables with integral coefficients. We replace the rational integers by the integers in a number field and examine the entropy of the corresponding dynamical system.

1. Introduction. We shall study the entropy of the following dynamical systems which arise from algebra and harmonical analysis. For every discrete $\mathbb{Z}\left[x_{1}^{ \pm 1}, \ldots, x_{d}^{ \pm 1}\right]$-module $M$ one has a corresponding compact dual group $X=\widehat{M}$ and an action of $\mathbb{Z}^{d}$ on $X$ which is given by the automorphism dual to multiplication by the monomials. In [4] one can find the following entropy formula for a special class of such dynamical systems which are building blocks for more complex ones. The entropy of the $\mathbb{Z}^{d}$-action $\alpha^{X}$ on the dual group $X$ of the cyclic module $M=\mathbb{Z}\left[x_{1}^{ \pm 1}, \ldots, x_{d}^{ \pm 1}\right] /(f)$ is given by

$$
\mathrm{h}\left(\alpha^{X}\right)= \begin{cases}\log \mathbb{M}(f) & \text { if } f \in \mathbb{Z}\left[x_{1}^{ \pm 1}, \ldots, x_{d}^{ \pm 1}\right] \backslash\{0\}, \\ \infty & \text { if } f=0\end{cases}
$$

where the Mahler measure $\mathbb{M}(f)$ of a nonzero polynomial $f \in \mathbb{Z}\left[x_{1}^{ \pm 1}, \ldots, x_{d}^{ \pm 1}\right]$ is given by

$$
\mathbb{M}(f)=\exp \left(\int_{\mathbb{T}^{d}} \log f(x) d x\right) .
$$

Here the integral is taken with respect to the normalised Haar measure of the $d$-dimensional torus.

We shall see that one gets an analogous result if one replaces the rational integers by the integers of a number field. Let $\mathbb{K}$ be a finite field extension of $\mathbb{Q}$ and let $\mathcal{O}_{\mathbb{K}}$ be the ring of integral elements of $\mathbb{K}$ over $\mathbb{Z}$. For a principal

1991 Mathematics Subject Classification: Primary 11R04, 28D15, 28 D20. 
ideal $(f) \neq 0$ in the $\operatorname{ring} \mathcal{O}_{\mathbb{K}}\left[x_{1}^{ \pm 1}, \ldots, x_{d}^{ \pm 1}\right]$ we prove the entropy formula

$$
\mathrm{h}\left(\alpha^{X}\right)=\log \mathbb{M}\left(N_{\mathbb{K} \mid \mathbb{Q}}(f)\right)
$$

for the dual group $X$ of the $\mathbb{Z}\left[x_{1}^{ \pm 1}, \ldots, x_{d}^{ \pm 1}\right]$-module

$$
\mathcal{O}_{\mathbb{K}}\left[x_{1}^{ \pm 1}, \ldots, x_{d}^{ \pm 1}\right] /(f) .
$$

The symbol $N_{\mathbb{K} \mid \mathbb{Q}}$ means the norm of the field $\mathbb{K}$ over $\mathbb{Q}$ extended to the corresponding rings of polynomials.

The module in (3) is cyclic as a $\mathcal{O}_{\mathbb{K}}\left[x_{1}^{ \pm 1}, \ldots, x_{d}^{ \pm 1}\right]$-module but not as a $\mathbb{Z}\left[x_{1}^{ \pm 1}, \ldots, x_{d}^{ \pm 1}\right]$-module, therefore the formula (1) is not applicable. One can of course build up this module by using cyclic $\mathbb{Z}\left[x_{1}^{ \pm 1}, \ldots, x_{d}^{ \pm 1}\right]$-modules and from this compute the entropy of the $\mathbb{Z}^{d}$-action. But the formula (2) uses the number-theoretical advantages of the module (3) and is easier for calculations.

We also study the entropy for nonprincipal ideals and for localisations of $\mathcal{O}_{\mathbb{K}}$.

I wish to thank Klaus Schmidt for many inspiring conversations, for looking over the text many times and for Remark 3.11. I also want to thank Johanna Gaier for helping me with my English.

2. Definitions. For any ring $A$ and any natural number $d$ we define the ring of polynomials

$$
L_{d}(A)=A\left[x_{1}^{ \pm 1}, \ldots, x_{d}^{ \pm 1}\right]
$$

in the commuting variables $x_{1}, \ldots, x_{d}$. We shall often write $\mathbf{x}^{\mathbf{n}}$ instead of $x_{1}^{n_{1}} \ldots x_{d}^{n_{d}}$. With this notation a typical polynomial $f \in L_{d}(A)$ has the form

$$
f=\sum_{\mathbf{n} \in \mathbb{Z}^{d}} c_{f}(\mathbf{n}) \mathbf{x}^{\mathbf{n}}
$$

where only finitely many coefficients $c_{f}(\mathbf{n}) \in A$ are nonzero.

Any ideal $I \subseteq L_{d}(A)$ defines an action of $\mathbb{Z}^{d}$ on the dual compact group $X=\left(L_{d}(A) / I\right)^{\wedge}$. The map $\alpha_{\mathbf{n}}^{X}$ is defined as the dual of multiplication by $\mathbf{x}^{\mathbf{n}}$. In the following we denote the entropy of this action by $\mathrm{h}_{A}(I)$. In the special case of a principal ideal $I=(f)_{A}$ we also write $\mathrm{h}_{A}(f)$.

Using Yuzvinskiıı's formula (see [4]) one can prove the additivity of $\mathrm{h}_{A}(\cdot)$, which means that $\mathrm{h}_{A}(f J)=\mathrm{h}_{A}(f)+\mathrm{h}_{A}(J)$ for any ideal $J \subseteq L_{d}(A)$ and any polynomial $f \in L_{d}(A)$.

Let $\mathbb{K} \mid \mathbb{Q}$ be a finite algebraic field extension with degree $n$ and let $\left\{\tau_{1}, \ldots, \tau_{n}\right\}$ be the complete set of field homomorphisms from $\mathbb{K}$ to the field $\mathbb{C}$ of complex numbers. For any polynomial $f \in L_{d}(\mathbb{K})$ we define the norm

$$
N_{\mathbb{K} \mid \mathbb{Q}}(f)=\prod_{i=1}^{n} \tau_{i}(f),
$$


where $\tau_{i}(f)$ is the polynomial over the complex field one gets by applying the homomorphism $\tau_{i}$ to the coefficients of $f$. If no misunderstandings are possible we also write $N(f)$ for the norm of $f$.

3. The entropy of $\left(L_{d}\left(\mathcal{O}_{\mathbb{K}}\right) /(f)\right)^{\wedge}$. We start our study of entropy with the special case of a principal ideal in the ring of algebraic integers of a finite normal field extension of $\mathbb{Q}$.

In order to get a connection between the entropies $h_{\mathbb{Z}}$ and $h_{\mathcal{O}_{\mathbb{K}}}$ we prove the following lemma.

3.1. Lemma. Let $\mathbb{L} \mid \mathbb{K}$ and $\mathbb{K} \mid \mathbb{Q}$ be finite algebraic field extensions and let $n=[\mathbb{L}: \mathbb{K}]$. For any principal ideal domain $A \subseteq \mathbb{K}$ which generates $\mathbb{K}$ as a field over $\mathbb{Q}$ and its integral closure $B$ in $L$ we have

$$
\mathrm{h}_{B}(f)=n \mathrm{~h}_{A}(f)
$$

for every polynomial $f \in L_{d}(A)$.

Proof. By the separability of $\mathbb{L} \mid \mathbb{K}$ there exists an integral basis $\left\{\alpha_{1}, \ldots, \alpha_{n}\right\}$ of $B$ over $A$ (see for example Theorem I.2.10 of [3]). We prove the lemma by defining an $L_{d}(A)$-module isomorphism

$$
\left(L_{d}(A) /(f)_{L_{d}(A)}\right)^{n} \cong L_{d}(B) /(f)_{L_{d}(B)} .
$$

Consider the well-defined morphism

$$
\Phi:\left(L_{d}(A) /(f)_{L_{d}(A)}\right)^{n} \rightarrow L_{d}(B) /(f)_{L_{d}(B)}
$$

given by $\Phi\left(\left(h_{i}+(f)_{L_{d}(A)}\right)_{i=1, \ldots, n}\right)=\sum_{i} h_{i} \alpha_{i}+(f)_{L_{d}(B)}$. Since every element of $B$ can be written as a linear combination of the $\alpha_{i}$ with coefficients in $A$, one can write every element of $L_{d}(B)$ as a linear combination of the $\alpha_{i}$ with coefficients in $L_{d}(A)$. It follows that $\Phi$ is surjective.

For the proof of the injectivity we assume that $\Phi\left(\left(h_{i}+(f)\right)_{i}\right)=0$, i.e., that there exist $h_{i}^{\prime}$ such that $\sum_{i} h_{i} \alpha_{i}=\left(\sum_{i} h_{i}^{\prime} \alpha_{i}\right) f$. The uniqueness of the above representation shows that $h_{i}=h_{i}^{\prime} f \equiv 0$. Therefore the morphism $\Phi$ is injective.

3.2. Proposition. Let $\mathbb{K} \mid \mathbb{Q}$ be a normal finite algebraic field extension and let $\mathcal{O}_{\mathbb{K}} \subseteq \mathbb{K}$ be the domain of algebraic integers in $\mathbb{K}$. The entropy of a nonzero polynomial $f \in \mathcal{O}_{\mathbb{K}}\left[x_{1}^{ \pm 1}, \ldots, x_{d}^{ \pm 1}\right]$ is given by

$$
\mathrm{h}_{\mathcal{O}_{\mathbb{K}}}(f)=\log \mathbb{M}(N(f)) \text {. }
$$

Proof. Let $\tau$ be a field automorphism of $\mathbb{K}$. Then the $L_{d}(\mathbb{Z})$-modules $L_{d}\left(\mathcal{O}_{\mathbb{K}}\right) /(f)$ and $L_{d}\left(\mathcal{O}_{\mathbb{K}}\right) /(\tau(f))$ are isomorphic by means of $\tau$. This proves that $\mathrm{h}_{\mathcal{O}_{\mathbb{K}}}(f)=\mathrm{h}_{\mathcal{O}_{\mathbb{K}}}(\tau(f))$. If $G=\left\{\tau_{1}, \ldots, \tau_{n}\right\}$ is the Galois group of $\mathbb{K}$ over $\mathbb{Q}$, then the addition formula mentioned in Section 2 and Lemma 3.1 imply that 


$$
\begin{aligned}
\mathrm{h}_{\mathcal{O}_{\mathbb{K}}}(f) & =\frac{\mathrm{h}_{\mathcal{O}_{\mathbb{K}}}\left(\tau_{1}(f)\right)+\ldots+\mathrm{h}_{\mathcal{O}_{\mathbb{K}}}\left(\tau_{n}(f)\right)}{n} \\
& =\frac{\mathrm{h}_{\mathcal{O}_{\mathbb{K}}}(N(f))}{n}=\mathrm{h}_{\mathbb{Z}}(N(f))=\log \mathbb{M}(N(f)) .
\end{aligned}
$$

3.3. ExAmple. For $\mathbb{K}=\mathbb{Q}(\sqrt{2})$ and $f=3 x+\sqrt{2} \in L_{1}(\mathbb{Z}[\sqrt{2}])$ we can easily compute the norm of $f$ :

$$
N(f)=(3 x+\sqrt{2})(3 x-\sqrt{2})=9 x^{2}-2 .
$$

The entropy of the $\mathbb{Z}$-action on the compact group

$$
\left(\mathbb{Z}\left[\sqrt{2}, x^{ \pm 1}\right] /(3 x+\sqrt{2})\right)^{\wedge}
$$

is $\log \mathbb{M}\left(9 x^{2}-2\right)=\log 9$, by Proposition 3.2.

As a generalisation of the greatest common divisor of the coefficients of a polynomial we define the content of a polynomial.

3.4. Definition. Let $A$ be a Dedekind domain with field of fractions $\mathbb{K}$ and let

$$
f=\sum_{\mathbf{m}} c_{f}(\mathbf{m}) \mathbf{x}^{\mathbf{m}} \in L_{d}(\mathbb{K})
$$

be a Laurent polynomial. We define the content of $f$ as the fractional ideal of $A$ generated by the coefficients of $f$ :

$$
\operatorname{Con}_{A}(f)=\left(c_{f}(\mathbf{m}): \mathbf{m} \in \mathbb{Z}^{d}\right)_{A} .
$$

A polynomial $f \in L_{d}(\mathbb{K})$ is called primitive if the content of $f$ is equal to $A$.

One form of Gauss' lemma in a unique factorization domain says that the content of polynomials is multiplicative. We now state the analogous statement for our domains and our definition of content. This can be found for instance as Satz 13 in Hilbert's report "Die Theorie der algebraischen Zahlkörper" (see [1], p. 78).

3.5. Lemma. Let $\mathcal{O}_{\mathbb{K}}$ be the domain of algebraic integers in a number field $\mathbb{K}$ and let $f, g \in L_{d}(\mathbb{K})$ be two Laurent polynomials. Then

$$
\operatorname{Con}_{\mathcal{O}_{\mathbb{K}}}(f g)=\operatorname{Con}_{\mathcal{O}_{\mathbb{K}}}(f) \operatorname{Con}_{\mathcal{O}_{\mathbb{K}}}(g)
$$

with the usual definition of ideal multiplication.

3.6. Lemma. Let $\mathbb{K} \mid \mathbb{Q}$ be a finite algebraic field extension, let $\mathcal{O}_{\mathbb{K}}$ be the domain of algebraic integers in $\mathbb{K}$ and let $f \in L_{d}\left(\mathcal{O}_{\mathbb{K}}\right)$ be a primitive polynomial. For each set $S \subseteq \mathcal{O}_{\mathbb{K}} \backslash\{0\}$ which is closed under multiplication we have

$$
\mathrm{h}_{\mathcal{O}_{\mathbb{K}}}(f)=\mathrm{h}_{S^{-1} \mathcal{O}_{\mathbb{K}}}(f),
$$

where $S^{-1} \mathcal{O}_{\mathbb{K}}$ denotes the localisation of $\mathcal{O}_{\mathbb{K}}$ with respect to the set $S$. 
Proof. We first prove that

$$
(f)_{L_{d}\left(S^{-1} \mathcal{O}_{\mathbb{K}}\right)} \cap L_{d}\left(\mathcal{O}_{\mathbb{K}}\right)=(f)_{L_{d}\left(\mathcal{O}_{\mathbb{K}}\right)} .
$$

Let $g \in L_{d}\left(S^{-1} \mathcal{O}_{\mathbb{K}}\right)$ with $g f \in L_{d}\left(\mathcal{O}_{\mathbb{K}}\right)$. Using Lemma 3.5 we get

$$
\operatorname{Con}_{\mathcal{O}_{\mathbb{K}}}(g)=\operatorname{Con}_{\mathcal{O}_{\mathbb{K}}}(g) \operatorname{Con}_{\mathcal{O}_{\mathbb{K}}}(f)=\operatorname{Con}_{\mathcal{O}_{\mathbb{K}}}(g f) \subseteq \mathcal{O}_{\mathbb{K}},
$$

so that $g$ lies in $L_{d}\left(\mathcal{O}_{\mathbb{K}}\right)$ and the above statement is true.

Let $s_{1}, s_{2}, \ldots$ be an enumeration of $S$. We define

$$
t_{n}= \begin{cases}1 & \text { if } n=0 \\ \prod_{i=1}^{n} s_{i} & \text { if } n>0\end{cases}
$$

For the sequence of $\mathbb{Z}$-modules $M_{n}=\left(1 / t_{n}\right) \mathcal{O}_{\mathbb{K}}$ we have $\bigcup_{n=1}^{\infty} M_{n}=S^{-1} \mathcal{O}_{\mathbb{K}}$, and the union of the $L_{d}(\mathbb{Z})$-modules

$$
\sum_{\mathbb{Z}^{d}} M_{n} /\left((f)_{L_{d}\left(S^{-1} \mathcal{O}_{\mathbb{K}}\right)} \cap \sum_{\mathbb{Z}^{d}} M_{n}\right)
$$

is equal to $L_{d}\left(S^{-1} \mathcal{O}_{\mathbb{K}}\right) /(f)_{L_{d}\left(S^{-1} \mathcal{O}_{\mathbb{K}}\right)}$, where we have identified the sets $\sum_{\mathbb{Z}^{d}} S^{-1} \mathcal{O}_{\mathbb{K}}$ and $L_{d}\left(S^{-1} \mathcal{O}_{\mathbb{K}}\right)$. Therefore, in the dual group

$$
X=(f)_{L_{d}\left(S^{-1} \mathcal{O}_{\mathbb{K}}\right)}^{\perp} \subseteq\left(\left(S^{-1} \mathcal{O}_{\mathbb{K}}\right)^{\wedge}\right)^{\mathbb{Z}^{d}}
$$

the intersection of the subgroups

$$
V_{n}=\left(\sum_{\mathbb{Z}^{d}} M_{n}\right)^{\perp}
$$

is $\{0\}$. By Lemma 13.6 of [4] we conclude that $\mathrm{h}\left(\sigma^{X / V_{n}}\right) \rightarrow \mathrm{h}\left(\sigma^{X}\right)$. The module

$$
\sum_{\mathbb{Z}^{d}} M_{n} /\left((f)_{L_{d}\left(S^{-1} \mathcal{O}_{\mathbb{K}}\right)} \cap \sum_{\mathbb{Z}^{d}} M_{n}\right),
$$

which is the dual group of $X / V_{n}$, is isomorphic to

$$
L_{d}\left(\mathcal{O}_{\mathbb{K}}\right) /\left((f)_{L_{d}\left(S^{-1} \mathcal{O}_{\mathbb{K}}\right)} \cap L_{d}\left(\mathcal{O}_{\mathbb{K}}\right)\right)=L_{d}\left(\mathcal{O}_{\mathbb{K}}\right) /(f)_{L_{d}\left(\mathcal{O}_{\mathbb{K}}\right)} .
$$

This is easily seen by using the morphism $\Phi(x)=t_{n} x$ from $\sum_{\mathbb{Z}^{d}} M_{n}$ to $L_{d}\left(\mathcal{O}_{\mathbb{K}}\right)$ which maps $(f)_{L_{d}\left(S^{-1} \mathcal{O}_{\mathbb{K}}\right)} \cap \sum_{\mathbb{Z}^{d}} M_{n}$ to $(f)_{L_{d}\left(S^{-1} \mathcal{O}_{\mathbb{K}}\right)} \cap L_{d}\left(\mathcal{O}_{\mathbb{K}}\right)$. Now the convergence of the entropies yields

$$
\mathrm{h}_{S^{-1} \mathcal{O}_{\mathbb{K}}}(f)=\mathrm{h}\left(\sigma^{X}\right)=\mathrm{h}_{\mathcal{O}_{\mathbb{K}}}(f) \text {. }
$$

3.7. EXAmple. We return to the field $\mathbb{K}=\mathbb{Q}(\sqrt{2})$ and the polynomial $f=3 x+\sqrt{2} \in L_{1}(\mathbb{Z}[\sqrt{2}])$ of Example 3.3. The polynomial $f$ is primitive and for each localisation with respect to a multiplicatively closed set $S \subseteq$ $\mathcal{O}_{\mathbb{K}} \backslash\{0\}$,

$$
\mathrm{h}_{S^{-1} \mathcal{O}_{\mathbb{K}}}(f)=\mathrm{h}_{\mathcal{O}_{\mathbb{K}}}(f)=\log 9 .
$$


3.8. Definition. Let $\mathbb{K} \mid \mathbb{Q}$ be a finite algebraic field extension and let $\mathcal{O}_{\mathbb{K}}$ be the domain of algebraic integers in $\mathbb{K}$. For each ideal $J \subseteq \mathcal{O}_{\mathbb{K}}$ we define its norm as $\mathcal{N}(J)=\left|\mathcal{O}_{\mathbb{K}} / J\right|$.

For principal ideals this definition coincides with the usual one (Section I.6 of [3]). We thus have

$$
\mathcal{N}((a))=\left|N_{\mathbb{K} \mid \mathbb{Q}}(a)\right| .
$$

3.9. Lemma. Let $\mathbb{K} \mid \mathbb{Q}$ be a finite algebraic field extension, let $\mathcal{O}_{\mathbb{K}}$ be the domain of integers in $\mathbb{K}$ and let $S \subseteq \mathcal{O}_{\mathbb{K}} \backslash\{0\}$ be closed under multiplication. For each nonzero prime ideal $\mathfrak{p} \subseteq \mathcal{O}_{\mathbb{K}}$ let $\|\cdot\|_{\mathfrak{p}}$ be the valuation such that $\|a\|_{\mathfrak{p}}=1 /\left|\mathcal{O}_{\mathbb{K}} / \mathfrak{p}\right|$ for every $a \in \mathfrak{p} \backslash \mathfrak{p}^{2}$. For each nonzero ideal $J \subseteq S^{-1} \mathcal{O}_{\mathbb{K}}$ we have the entropy formula

$$
\mathrm{h}_{S^{-1} \mathcal{O}_{\mathbb{K}}}(J)=\log \left(\mathcal{N}\left(J \cap \mathcal{O}_{\mathbb{K}}\right)\right),
$$

and for every nonzero element $a \in S^{-1} \mathcal{O}_{\mathbb{K}}$,

$$
\mathrm{h}_{S^{-1} \mathcal{O}_{\mathbb{K}}}(a)=\log \left(\left|N_{\mathbb{K} \mid \mathbb{Q}}(a)\right| \prod_{\mathfrak{p} \cap S \neq \emptyset}\|a\|_{\mathfrak{p}}\right) .
$$

Proof. The Chinese remainder theorem (see [3], I.3.6) and the ring isomorphism $\mathcal{O}_{\mathbb{K}} / \mathfrak{p}^{n} \cong \mathcal{O}_{\mathbb{K}, \mathfrak{p}} /\left(\mathfrak{p} \mathcal{O}_{\mathbb{K}, \mathfrak{p}}\right)^{n}$ (see [3], I.11.2) show that

$$
\begin{aligned}
S^{-1} \mathcal{O}_{\mathbb{K}} / J & \cong \mathcal{O}_{\mathbb{K}, \mathfrak{p}_{1}} /\left(\mathfrak{p}_{1} \mathcal{O}_{\mathbb{K}, \mathfrak{p}_{1}}\right)^{n_{1}} \oplus \ldots \oplus \mathcal{O}_{\mathbb{K}, \mathfrak{p}_{r}} /\left(\mathfrak{p}_{r} \mathcal{O}_{\mathbb{K}, \mathfrak{p}_{r}}\right)^{n_{r}} \\
& \cong \mathcal{O}_{\mathbb{K}} /\left(J \cap \mathcal{O}_{\mathbb{K}}\right),
\end{aligned}
$$

where $J \cap \mathcal{O}_{\mathbb{K}}$ has the prime decomposition $\mathfrak{p}_{1}^{n_{1}} \ldots \mathfrak{p}_{r}^{n_{r}}$. The last term in (4) has exactly $\mathcal{N}\left(J \cap \mathcal{O}_{\mathbb{K}}\right)$ elements. The Haar measure of

$$
\left(\left(\mathcal{O}_{\mathbb{K}} /\left(J \cap \mathcal{O}_{\mathbb{K}}\right)\right)^{\wedge}\right)^{\mathbb{Z}^{d}} \cong\left(L_{d}\left(S^{-1} \mathcal{O}_{\mathbb{K}}\right) /\left(J L_{d}\left(S^{-1} \mathcal{O}_{\mathbb{K}}\right)\right)\right)^{\wedge}
$$

is the uniformly distributed product measure. The first statement of the lemma follows from the well known entropy formula for full shifts.

For the second statement we have to determine the number of elements of $S^{-1} \mathcal{O}_{\mathbb{K}} /(a)$. The Chinese remainder theorem yields

$$
S^{-1} \mathcal{O}_{\mathbb{K}} /(a) \cong \mathcal{O}_{\mathbb{K}, \mathfrak{p}_{1}} /\left(\mathfrak{p}_{1} \mathcal{O}_{\mathbb{K}, \mathfrak{p}_{1}}\right)^{n_{1}} \oplus \ldots \oplus \mathcal{O}_{\mathbb{K}, \mathfrak{p}_{r}} /\left(\mathfrak{p}_{r} \mathcal{O}_{\mathbb{K}, \mathfrak{p}_{r}}\right)^{n_{r}},
$$

where the $\mathfrak{p}_{i}$ are those prime ideals which are factors of $a$ disjoint from $S$. Therefore the number of elements is

$$
\frac{1}{\prod_{\mathfrak{p} \cap S=\emptyset}\|a\|_{\mathfrak{p}}}=\left|N_{\mathbb{K} \mid \mathbb{Q}}(a)\right| \prod_{\mathfrak{p} \cap S \neq \emptyset}\|a\|_{\mathfrak{p}},
$$

where we have used the product formula (see [3], Satz III.1.3).

3.10. TheOREM. Let $\mathbb{K} \mid \mathbb{Q}$ be an algebraic finite field extension and let $\mathcal{O}_{\mathbb{K}} \subseteq \mathbb{K}$ be the corresponding domain of algebraic integers. For each nonzero 
Laurent polynomial $f \in L_{d}\left(\mathcal{O}_{\mathbb{K}}\right)$,

$$
\mathrm{h}_{\mathcal{O}_{\mathbb{K}}}(f)=\log \mathbb{M}(N(f)) \text {. }
$$

Proof. We start with the case of a primitive polynomial. We apply Lemma 3.6 to $S=\mathcal{O}_{\mathbb{K}} \backslash\{0\}$ to get

$$
\mathrm{h}_{\mathcal{O}_{\mathbb{K}}}(f)=\mathrm{h}_{\mathbb{K}}(f) \text {. }
$$

Let $\mathbb{L} \mid \mathbb{K}$ be a normal extension over $\mathbb{Q}$ of degree $[\mathbb{L}: \mathbb{K}]=l$. Lemma 3.1 with $A=\mathbb{K}$ and $B=\mathbb{L}$ yields

$$
\mathrm{h}_{\mathbb{K}}(f)=\frac{1}{l} \mathrm{~h}_{\mathbb{L}}(f) .
$$

Since $f$ is primitive as an element of $L_{d}\left(\mathcal{O}_{\mathbb{K}}\right)$, one can write the identity in $\mathcal{O}_{\mathbb{K}}$ as a linear combination of the coefficients of $f$, which is a representation of the identity with values in $\mathcal{O}_{\mathbb{L}}$. Therefore $f$ is also primitive as an element of $L_{d}\left(\mathcal{O}_{\mathbb{L}}\right)$. By Lemma 3.6 one gets

$$
\mathrm{h}_{\mathcal{O}_{\mathbb{L}}}(f)=\mathrm{h}_{\mathbb{L}}(f) .
$$

Together with Proposition 3.2 we have

$$
\mathrm{h}_{\mathcal{O}_{\mathbb{K}}}(f)=\frac{1}{l}\left|\log \mathbb{M}\left(N_{\mathbb{L} \mid \mathbb{Q}}(f)\right)\right|=\left|\log \mathbb{M}\left(N_{\mathbb{K} \mid \mathbb{Q}}(f)\right)\right| .
$$

If $f$ is not primitive then we can find a power of $f$ such that the content of $f^{m}$ is a principal ideal $(a)$ :

$$
\operatorname{Con}\left(f^{m}\right)=\operatorname{Con}(f)^{m}=(a)
$$

by Lemma 3.5 and the fact that the ideal class group has finite order. Now from the first part of the proof we get

$$
\mathrm{h}_{\mathcal{O}_{\mathbb{K}}}\left(\frac{1}{a} f^{m}\right)=\left|\log \mathbb{M}\left(N_{\mathbb{K} \mid \mathbb{Q}}\left(\frac{1}{a} f^{m}\right)\right)\right| .
$$

By Lemma 3.9 we see that

$$
\mathrm{h}_{\mathcal{O}_{\mathbb{K}}}(a)=|\log | N_{\mathbb{K} \mid \mathbb{Q}}(a)||=\left|\log \mathbb{M}\left(N_{\mathbb{K} \mid \mathbb{Q}}(a)\right)\right| .
$$

The transformations

$$
\begin{aligned}
\mathrm{h}_{\mathcal{O}_{\mathbb{K}}}(f) & =\frac{1}{m}\left(\mathrm{~h}_{\mathcal{O}_{\mathbb{K}}}(a)+\mathrm{h}_{\mathcal{O}_{\mathbb{K}}}\left(\frac{1}{a} f^{m}\right)\right) \\
& =\frac{1}{m}\left(\log \mathbb{M}\left(N_{\mathbb{K} \mid \mathbb{Q}}(a)\right)+\log \mathbb{M}\left(N_{\mathbb{K} \mid \mathbb{Q}}\left(\frac{1}{a} f^{m}\right)\right)\right) \\
& =\frac{1}{m} \log \mathbb{M}\left(N_{\mathbb{K} \mid \mathbb{Q}}\left(f^{m}\right)\right)=\log \mathbb{M}\left(N_{\mathbb{K} \mid \mathbb{Q}}(f)\right)
\end{aligned}
$$

conclude the proof.

3.11. Remark. Theorem 3.10 can be proved more quickly if one uses Example 18.7 of [4]: the entropy of the $\mathbb{Z}^{d}$-shift on the dual group of the 
$L_{d}(\mathbb{Z})$-module $L_{d}(\mathbb{Z})^{n} / A L_{d}(\mathbb{Z})^{n}$ for a matrix $A \in \operatorname{Mat}_{n}\left(L_{d}(\mathbb{Z})\right)$ is given by $|\log (\mathbb{M}(\operatorname{det}(A)))|$.

As we have already seen in the proof of Lemma 3.1 the $L_{d}(\mathbb{Z})$-module $L_{d}\left(\mathcal{O}_{\mathbb{K}}\right)$ is isomorphic to $L_{d}(\mathbb{Z})^{n}$. Multiplication by $f$ is an $L_{d}(\mathbb{Z})$-linear map and is therefore represented by a matrix $A_{f} \in \operatorname{Mat}_{n}\left(L_{d}(\mathbb{Z})\right)$. For the proof of Theorem 3.10 one only has to check the formula

$$
N_{\mathbb{K} \mid \mathbb{Q}}(f)=\operatorname{det}\left(A_{f}\right),
$$

which can be deduced in a way analogous to the corresponding equality for numbers (see for example Section I.2 in [3]).

3.12. ExAmple. Let $\mathbb{K}=\mathbb{Q}[\sqrt[3]{2}]$ and $f=2 x^{2}+\sqrt[3]{2}$. Then

$$
N(f)=\left(2 x^{2}+\sqrt[3]{2}\right)\left(2 x^{2}+\zeta \sqrt[3]{2}\right)\left(2 x^{2}+\zeta^{2} \sqrt[3]{2}\right)=8 x^{6}+2,
$$

where $\zeta$ denotes a primitive third root of the unity. Now one can compute the entropy from Theorem 3.10 as follows:

$$
\mathrm{h}_{\mathcal{O}_{\mathbb{K}}}(f)=\log \mathbb{M}\left(8 x^{6}+2\right)=\log 8 .
$$

3.13. Corollary. Let $\mathbb{K} \mid \mathbb{Q}$ be a finite algebraic field extension, let $\mathcal{O}_{\mathbb{K}} \subseteq$ $\mathbb{K}$ be the domain of algebraic integers and let $S \subseteq \mathcal{O}_{\mathbb{K}} \backslash\{0\}$ be closed under multiplication. For each prime ideal $\mathfrak{p}$ let $\|\cdot\|_{\mathfrak{p}}$ be the valuation such that $\|a\|_{\mathfrak{p}}=1 /\left|\mathcal{O}_{\mathbb{K}} / \mathfrak{p}\right|$ for every $a \in \mathfrak{p} \backslash \mathfrak{p}^{2}$. For every nonzero polynomial $f \in$ $L_{d}\left(S^{-1} \mathcal{O}_{\mathbb{K}}\right)$ we have

$$
\mathrm{h}_{S^{-1} \mathcal{O}_{\mathbb{K}}}(f)=\log \left(\mathbb{M}\left(N_{\mathbb{K} \mid \mathbb{Q}}(f)\right) \prod_{\mathfrak{p} \cap S \neq \emptyset}\left\|\operatorname{Con}_{\mathcal{O}_{\mathbb{K}}}(f)\right\|_{\mathfrak{p}}\right) .
$$

Pr o of. For a primitive polynomial we argue as for Theorem 3.10.

For the general case we also examine the polynomial $f^{m}$. For the generator $a$ of the content of $f^{m}$ we know the entropy from Lemma 3.9:

$$
\mathrm{h}_{S^{-1} \mathcal{O}_{\mathbb{K}}}(a)=\log \left(\mathbb{M}\left(N_{\mathbb{K} \mid \mathbb{Q}}(a)\right) \prod_{\mathfrak{p} \cap S \neq \emptyset}\|a\|_{\mathfrak{p}}\right) .
$$

The polynomial $(1 / a) f^{m}$ is primitive and we conclude the proof in the same way as for Theorem 3.10.

3.14. EXAmPLE. Let $K=\mathbb{Q}(\sqrt{2})$ and

$$
f=(3+\sqrt{2}) x-7=(3+\sqrt{2})(x-(3-\sqrt{2})) .
$$

Then

$$
N(f)=7\left(x^{2}-7\right)
$$

We choose $S=\left\{7^{n}: n \in \mathbb{N}\right\}$. The content of $f$ is $\operatorname{Con}_{\mathcal{O}_{\mathbb{K}}}(f)=(3+\sqrt{2})$, and the prime decomposition of 7 in the unique factorisation domain $\mathbb{Z}[\sqrt{2}]$ has the form

$$
7=(3+\sqrt{2})(3-\sqrt{2}) .
$$


Using Corollary 3.13 we get

$$
\mathrm{h}_{\mathbb{Z}[\sqrt{2}, 1 / 7]}(f)=\log \left(\mathbb{M}(N(f)) \prod_{\mathfrak{p} \cap S \neq\{0\}}\|\operatorname{Con}(f)\|_{\mathfrak{p}}\right)=\log \left(49 \cdot \frac{1}{7}\right)=\log 7 .
$$

4. The entropy of $\left(L_{d}\left(\mathcal{O}_{\mathbb{K}}\right) / J\right)^{\wedge}$. Now we start our examination of nonprincipal ideals $J \subseteq L_{d}\left(S^{-1} \mathcal{O}_{\mathbb{K}}\right)$ and prove an entropy formula for the corresponding dynamical systems.

But first we prove some preliminary results.

4.1. Lemma. Let $\mathbb{K} \mid \mathbb{Q}$ be a finite algebraic field extension and let $\mathcal{O}_{\mathbb{K}}$ be its domain of algebraic integers. A polynomial $f \in L_{d}(\mathbb{Z})$ is primitive as an element of $L_{d}(\mathbb{Z})$ if and only if it is primitive as an element of $L_{d}\left(\mathcal{O}_{\mathbb{K}}\right)$. For $f \in L_{d}\left(\mathcal{O}_{\mathbb{K}}\right)$ we have $N(f) \in(f)_{L_{d}\left(\mathcal{O}_{\mathbb{K}}\right)} \cap L_{d}(\mathbb{Z})$. If the polynomial $f$ is also primitive, then the norm $N(f)$ is primitive.

Proof. For a primitive polynomial $f \in L_{d}(\mathbb{Z})$ all the coefficients are integers and the unity can be written as an integral combination of the coefficients. This representation is also a representation of the unity in $\mathcal{O}_{\mathbb{K}}$, so that $f$ is primitive as an element of $L_{d}\left(\mathcal{O}_{\mathbb{K}}\right)$.

If $f \in L_{d}(\mathbb{Z})$ is primitive with respect to $\mathcal{O}_{\mathbb{K}}$ but not with respect to $\mathbb{Z}$, then there exists a prime $p \in \mathbb{Z}$ which divides all the coefficients of $f$. We get $\operatorname{Con}_{\mathcal{O}_{\mathbb{K}}}(f) \subseteq p \mathcal{O}_{\mathbb{K}} \neq \mathcal{O}_{\mathbb{K}}$, contrary to the assumption that $f$ is primitive.

For the second statement of the lemma we have to prove that

$$
\frac{N_{\mathbb{K} \mid \mathbb{Q}}(f)}{f}=\prod_{\substack{\tau: \mathbb{K} \rightarrow \mathbb{L} \\ \tau \neq \mathrm{id}}} \tau(f) \in L_{d}\left(\mathcal{O}_{\mathbb{K}}\right),
$$

where $\mathbb{L}$ is a normal closure of $\mathbb{K}$ over $\mathbb{Q}$ and $\tau: \mathbb{K} \rightarrow \mathbb{L}$ are the possible field homomorphisms. The polynomial $N_{\mathbb{K} \mid \mathbb{Q}}(f) / f$ has coefficients in $\mathcal{O}_{\mathbb{L}}$. Since $f, N_{\mathbb{K} \mid \mathbb{Q}}(f) \in L_{d}(\mathbb{K})$ the quotient $N_{\mathbb{K} \mid \mathbb{Q}}(f) / f$ also has to be in $L_{d}(\mathbb{K})$. We conclude that $N_{\mathbb{K} \mid \mathbb{Q}}(f) / f \in L_{d}\left(\mathcal{O}_{\mathbb{K}}\right)$.

For the last statement we take a primitive polynomial $f \in L_{d}\left(\mathcal{O}_{\mathbb{K}}\right)$. As the unity is an integral combination of the coefficients of $f$, for any field homomorphism $\tau: \mathbb{K} \rightarrow \mathbb{L}$ the polynomial $\tau(f)$ is primitive in $L_{d}\left(\mathcal{O}_{\mathbb{L}}\right)$. Using Lemma 3.5 we see that

$$
N_{\mathbb{K} \mid \mathbb{Q}}(f)=\prod_{\tau: \mathbb{K} \rightarrow \mathbb{L}} \tau(f)
$$

is primitive in $L_{d}\left(\mathcal{O}_{\mathbb{L}}\right)$ and the first part of the lemma shows that $N(f)$ is primitive as an element of $L_{d}(\mathbb{Z})$.

Analogously to the content of a polynomial we need the notion of the content of an ideal. 
4.2. Definition. Let $A$ be a Dedekind domain with field of fractions $\mathbb{K}$ and let $J \subseteq L_{d}(\mathbb{K})$ be a finitely generated $L_{d}(A)$-submodule. We define the content of $J$ over $A$ as

$$
\operatorname{Con}_{A}(J)=\sum_{f \in J} \operatorname{Con}_{A}(f),
$$

and call an ideal $J$ primitive if its content is equal to $A$.

As an easy consequence of our definition and our form of Gauss' lemma (see Lemma 3.5) we can prove the following result.

4.3. Lemma. Let $\mathcal{O}_{\mathbb{K}}$ be the domain of integral elements in a number field $\mathbb{K}$ and let $J \subseteq L_{d}(\mathbb{K})$ be a finitely generated $L_{d}\left(\mathcal{O}_{\mathbb{K}}\right)$-submodule. There exists a polynomial $f \in J$ with

$$
\operatorname{Con}_{\mathcal{O}_{\mathbb{K}}}(J)=\operatorname{Con}_{\mathcal{O}_{\mathbb{K}}}(f) .
$$

For any $g \in L_{d}(\mathbb{K})$ we have

$$
\operatorname{Con}_{\mathcal{O}_{\mathbb{K}}}(g J)=\operatorname{Con}_{\mathcal{O}_{\mathbb{K}}}(g) \operatorname{Con}_{\mathcal{O}_{\mathbb{K}}}(J) .
$$

Proof. There exist polynomials $f_{1}, \ldots, f_{l} \in J$ whose coefficients generate $\operatorname{Con}_{\mathcal{O}_{\mathbb{K}}}(J)$. One can easily find a sequence $n_{2}, \ldots, n_{l}$ such that

$$
f=f_{1}+x_{1}^{n_{2}} f_{2}+\ldots+x_{1}^{n_{l}} f_{l} \in J
$$

has all the coefficients of $f_{1}, \ldots, f_{l}$ as coefficients. This means $\operatorname{Con}_{\mathcal{O}_{\mathbb{K}}}(f)=$ $\operatorname{Con}_{\mathcal{O}_{\mathbb{K}}}(J)$.

For $g \in L_{d}(\mathbb{K})$ and any $h \in J$ we now have, by Lemma 3.5,

$$
\begin{aligned}
\operatorname{Con}_{\mathcal{O}_{\mathbb{K}}}(g h) & =\operatorname{Con}_{\mathcal{O}_{\mathbb{K}}}(g) \operatorname{Con}_{\mathcal{O}_{\mathbb{K}}}(h) \subseteq \operatorname{Con}_{\mathcal{O}_{\mathbb{K}}}(g) \operatorname{Con}_{\mathcal{O}_{\mathbb{K}}}(J) \\
& =\operatorname{Con}_{\mathcal{O}_{\mathbb{K}}}(g) \operatorname{Con}_{\mathcal{O}_{\mathbb{K}}}(f)=\operatorname{Con}_{\mathcal{O}_{\mathbb{K}}}(g f) \subseteq \operatorname{Con}_{\mathcal{O}_{\mathbb{K}}}(g J)
\end{aligned}
$$

and therefore $\operatorname{Con}_{\mathcal{O}_{\mathbb{K}}}(g J)=\operatorname{Con}_{\mathcal{O}_{\mathbb{K}}}(g) \operatorname{Con}_{\mathcal{O}_{\mathbb{K}}}(J)$.

4.4. Lemma. Let $\mathbb{K} \mid \mathbb{Q}$ be a finite algebraic field extension, let $\mathcal{O}_{\mathbb{K}} \subseteq \mathbb{K}$ be the corresponding domain of integral elements and let $S \subseteq \mathcal{O}_{\mathbb{K}} \backslash\{0\}$ be closed under multiplication. For any ideal $I \subseteq L_{d}\left(S^{-1} \mathcal{O}_{\mathbb{K}}\right)$ which contains a primitive polynomial $f \in I$ and a nonzero constant $a \in I \cap S^{-1} \mathcal{O}_{\mathbb{K}}$, the entropy of the shift on $\left(L_{d}\left(S^{-1} \mathcal{O}_{\mathbb{K}}\right) / I\right)^{\wedge}$ vanishes.

Proof. The norms $N(f) \in L_{d}(\mathbb{Z})$ and $N(a) \in \mathbb{Z}$ are also elements of $I$, which was proved in Lemma 4.1. Therefore we can assume that $f \in I \cap L_{d}(\mathbb{Z})$ and $n \in I \cap \mathbb{Z}$. If we prove the statement for the ideal $I \cap L_{d}\left(\mathcal{O}_{\mathbb{K}}\right) \subseteq L_{d}\left(\mathcal{O}_{\mathbb{K}}\right)$ we also get it for $I \subseteq L_{d}\left(S^{-1} \mathcal{O}_{\mathbb{K}}\right)$ by a similar consideration to the proof of Lemma 3.6. The $L_{d}(\mathbb{Z})$-module isomorphism

$$
L_{d}\left(\mathcal{O}_{\mathbb{K}}\right) /(n, f)_{L_{d}\left(\mathcal{O}_{\mathbb{K}}\right)} \cong\left(L_{d}(\mathbb{Z}) /(n, f)_{L_{d}(\mathbb{Z})}\right)^{n},
$$


which can be seen as in the proof of Lemma 3.1, and the fact that $(n, f)_{L_{d}\left(\mathcal{O}_{\mathbb{K}}\right)}$ $\subseteq I \cap L_{d}\left(\mathcal{O}_{\mathbb{K}}\right)$ prove the lemma because the entropy of the $L_{d}(\mathbb{Z})$-ideal $(n, f)$ vanishes.

We have already defined the quantity $\mathrm{h}_{R}(\cdot)$ for a ring $R$. If $R$ is a unique factorisation domain, then so is the polynomial ring $L_{d}(R)$. Therefore each subset $A \subseteq L_{d}(R)$ admits a greatest common divisor $\operatorname{gcd}(A)$. In the next lemma we prove a connection between the entropy of the ideal and its greatest common divisor.

4.5. Lemma. Let $R$ be a Noetherian unique factorisation domain which satisfies $\mathrm{h}_{R}(f)<\infty$ for each nonzero $f \in L_{d}(R)$. Then for any nonzero ideal $J \subseteq L_{d}(R)$,

$$
\mathrm{h}_{R}(J)=\mathrm{h}_{R}(\operatorname{gcd}(J)) .
$$

Proof. First we remark that the lemma is equivalent to the special case of a primitive ideal, i.e., to the case where $\operatorname{gcd}(J)=1$. Let $J$ be an arbitrary ideal and denote by $g=\operatorname{gcd}(J)$ its common divisor. The ideal $g^{-1} J=\left\{f \in L_{d}(R): g f \in J\right\}$ satisfies $\operatorname{gcd}\left(g^{-1} J\right)=1$. Since $g\left(g^{-1} J\right)=J$, by additivity we have $\mathrm{h}_{R}(J)=\mathrm{h}_{R}(g)+\mathrm{h}_{R}\left(g^{-1} J\right)$.

We proceed by induction on the number of generators $f_{1}, \ldots, f_{l}$ of $J=$ $\left\langle f_{1}, \ldots, f_{l}\right\rangle$. We start with $l=2$ and $f=f_{1} \perp g=f_{2}$, and get the exact sequence

$$
0 \rightarrow L_{d}(R) /(f) \stackrel{* g}{\longrightarrow} L_{d}(R) /(f) \stackrel{\pi}{\rightarrow} L_{d}(R) /(f, g) \rightarrow 0,
$$

where the map $* g$ is multiplication by the polynomial $g$, and $\pi$ is the canonical projection. Since $f \perp g$, multiplication by $g$ is injective. The equation $\operatorname{im}(* g)=\operatorname{ker}(\pi)$ and the surjectivity of $\pi$ are also clear. As the entropy $\mathrm{h}_{R}(f)$ for $f \neq 0$ is finite we conclude by using the dual sequence that $\mathrm{h}_{R}((f, g))=0$.

Assume now that the lemma has already been proved for $l \geq 2$ generators. For a primitive ideal with $l+1$ generators $f_{1}, \ldots, f_{l+1}$ we define $g=\operatorname{gcd}\left(f_{1}, \ldots, f_{l}\right)$ and get an exact sequence

$$
\begin{aligned}
0 \rightarrow\left(\frac{f_{1}}{g}, \ldots, \frac{f_{l}}{g}, f_{l+1}\right) /\left(f_{1}, \ldots, f_{l+1}\right) & \rightarrow L_{d}(R) /\left(f_{1}, \ldots, f_{l+1}\right) \\
& \stackrel{* g}{\longrightarrow} L_{d}(R) /\left(f_{1}, \ldots, f_{l+1}\right) \stackrel{\pi}{\rightarrow} L_{d}(R) /\left(g, f_{l+1}\right) \rightarrow 0,
\end{aligned}
$$

which is easily proved as above.

The entropies of the corresponding dual groups are finite and therefore

$$
\begin{aligned}
\mathrm{h}_{R}\left(\left(g, f_{l+1}\right)\right)-\mathrm{h}_{R}\left(\left(f_{1}, \ldots, f_{l+1}\right)\right)+\mathrm{h}_{R}\left(\left(f_{1}, \ldots, f_{l+1}\right)\right) & \\
-\mathrm{h}\left(\sigma^{\left(\left(f_{1} / g, \ldots, f_{l} / g, f_{l+1}\right) /\left(f_{1}, \ldots, f_{l+1}\right)\right)^{\wedge}}\right) & =0 .
\end{aligned}
$$


The first expression above vanishes because of $g \perp f_{l+1}$ and the case $l=2$ which we have already proved. So we get

$$
\mathrm{h}\left(\sigma^{\left(\left(f_{1} / g, \ldots, f_{l} / g, f_{l+1}\right) /\left(f_{1}, \ldots, f_{l+1}\right)\right)^{\wedge}}\right)=0 .
$$

By the inductive assumption for the ideal $\left(f_{1} / g, \ldots, f_{l} / g\right)$ and the sequence

$$
L_{d}(R) /\left(\frac{f_{1}}{g}, \ldots, \frac{f_{l}}{g}\right) \rightarrow L_{d}(R) /\left(\frac{f_{1}}{g}, \ldots, \frac{f_{l}}{g}, f_{l+1}\right) \rightarrow 0,
$$

we have $\mathrm{h}\left(\sigma^{\left(L_{d}(R) /\left(f_{1} / g, \ldots, f_{l} / g, f_{l+1}\right)\right)^{\wedge}}\right)=0$. The exact sequence

$$
\begin{aligned}
0 \rightarrow\left(\frac{f_{1}}{g}, \ldots\right. & \left., \frac{f_{l}}{g}, f_{l+1}\right) /\left(f_{1}, \ldots, f_{l+1}\right) \\
& \rightarrow L_{d}(R) /\left(f_{1}, \ldots, f_{l+1}\right) \rightarrow L_{d}(R) /\left(\frac{f_{1}}{g}, \ldots, \frac{f_{l}}{g}, f_{l+1}\right) \rightarrow 0
\end{aligned}
$$

proves the induction step $\mathrm{h}_{R}\left(\left(f_{1}, \ldots, f_{l+1}\right)\right)=0$.

Now we can prove the generalisation of the entropy formula for nonprincipal ideals.

4.6. Theorem. Let $\mathbb{K} \mid \mathbb{Q}$ be a finite algebraic field extension of $\mathbb{Q}$, let $\mathcal{O}_{\mathbb{K}} \subseteq \mathbb{K}$ be the domain of integral elements in $\mathbb{K}$ and let $S \subseteq \mathcal{O}_{\mathbb{K}} \backslash\{0\}$ be closed under multiplication. For any nonzero ideal $J \subseteq L_{d}\left(S^{-1} \mathcal{O}_{\mathbb{K}}\right)$ we have the entropy formula

$$
\mathrm{h}_{S^{-1} \mathcal{O}_{\mathbb{K}}}(J)=\log \left(\mathbb{M}\left(\operatorname{pr} N\left(\operatorname{gcd}_{L_{d}(\mathbb{K})}(J)\right)\right) \mathcal{N}\left(\operatorname{Con}_{S^{-1} \mathcal{O}_{\mathbb{K}}}(J) \cap \mathcal{O}_{\mathbb{K}}\right)\right),
$$

where $\operatorname{pr} N(\cdot)$ denotes a primitive version of the norm of the polynomial.

Proof. We start with a new formulation of the entropy formula for a principal ideal $(g)$. Corollary 3.13 shows that the entropy is the logarithm of

$$
\mathbb{M}(N(g)) \prod_{\mathfrak{p} \cap S \neq \emptyset}\left\|\operatorname{Con}_{\mathcal{O}_{\mathbb{K}}}(g)\right\|_{\mathfrak{p}}=\mathbb{M}(\operatorname{pr} N(g)) \operatorname{Con}_{\mathbb{Z}}(N(g)) \prod_{\mathfrak{p} \cap S \neq \emptyset}\left\|\operatorname{Con}_{\mathcal{O}_{\mathbb{K}}}(g)\right\|_{\mathfrak{p}},
$$

where we identify the ideals in $\mathbb{Z}$ with their positive generators.

For the proof of the equality

$$
\operatorname{Con}_{\mathbb{Z}}(N(g))=\mathcal{N}\left(\operatorname{Con}_{\mathcal{O}_{\mathbb{K}}}(g)\right)
$$

we use as previously the polynomial $g^{m}$, where $m$ is a positive integer such that the content $\operatorname{Con}_{\mathcal{O}_{\mathbb{K}}}\left(g^{m}\right)=(a)$ is a principal ideal. From Gauss' lemma (see Lemma 3.5) we get

$$
\operatorname{Con}_{\mathbb{Z}}(N(g))=\sqrt[m]{\operatorname{Con}_{\mathbb{Z}}\left(N\left(g^{m}\right)\right)}=\sqrt[m]{\operatorname{Con}_{\mathbb{Z}}\left(N\left(\frac{1}{a} g^{m}\right)\right)|N(a)|}=\sqrt[m]{|N(a)|},
$$

because the norm of the primitive polynomial $g^{m} / a$ is primitive as we have seen in Lemma 4.1. 
On the right-hand side of (6) we make the same transformations to get

$$
\begin{aligned}
\mathcal{N}\left(\operatorname{Con}_{\mathcal{O}_{\mathbb{K}}}(g)\right) & =\sqrt[m]{\mathcal{N}\left(\operatorname{Con}_{\mathcal{O}_{\mathbb{K}}}\left(g^{m}\right)\right)} \\
& =\sqrt[m]{\mathcal{N}\left(\operatorname{Con}_{\mathcal{O}_{\mathbb{K}}}\left(\frac{1}{a} g^{m}\right)\right) \mathcal{N}(a)}=\sqrt[m]{|N(a)|}
\end{aligned}
$$

Therefore the entropy is the logarithm of

$$
\mathbb{M}(\operatorname{pr} N(g)) \mathcal{N}\left(\operatorname{Con}_{\mathcal{O}_{\mathbb{K}}}(g)\right) \prod_{\mathfrak{p} \cap S \neq \emptyset}\left\|\operatorname{Con}_{\mathcal{O}_{\mathbb{K}}}(g)\right\|_{\mathfrak{p}} .
$$

In order to prove the theorem for the case of a principal ideal we thus only have to show that

$$
\mathcal{N}\left(\operatorname{Con}_{\mathcal{O}_{\mathbb{K}}}(g)\right) \prod_{\mathfrak{p} \cap S \neq \emptyset}\left\|\operatorname{Con}_{\mathcal{O}_{\mathbb{K}}}(g)\right\|_{\mathfrak{p}}=\mathcal{N}\left(\operatorname{Con}_{S^{-1} \mathcal{O}_{\mathbb{K}}}(g) \cap \mathcal{O}_{\mathbb{K}}\right) .
$$

The proof of the above equality is analogous to a part of the proof of Lemma 3.9, where the Chinese remainder theorem was used.

For a nonprincipal ideal $J \subseteq L_{d}\left(S^{-1} \mathcal{O}_{\mathbb{K}}\right)$ we can define the inverted content

$$
I=\operatorname{Con}_{S^{-1} \mathcal{O}_{\mathbb{K}}}(J)^{-1}=\left\{a \in \mathbb{K}: a \operatorname{Con}_{S^{-1}} \mathcal{O}_{\mathbb{K}}(J) \subseteq S^{-1} \mathcal{O}_{\mathbb{K}}\right\}
$$

and define a finite set $\left\{\alpha_{1}, \ldots, \alpha_{r}\right\} \subseteq \mathbb{K}$ of generators of $I$. The content of the polynomial $g=\sum_{i=1}^{r} \alpha_{i} x_{1}^{i} \in L_{d}(\mathbb{K})$ is equal to $I$. By Lemma 4.3 the ideal $g J$ is primitive in $L_{d}\left(S^{-1} \mathcal{O}_{\mathbb{K}}\right)$. There exists an $a \in S^{-1} \mathcal{O}_{\mathbb{K}}$ with $g^{\prime}=a g \in L_{d}\left(S^{-1} \mathcal{O}_{\mathbb{K}}\right)$. With the two possibilities of writing the ideal agJ as a product of a polynomial or a constant with an ideal we get

$$
\mathrm{h}_{S^{-1} \mathcal{O}_{\mathbb{K}}}\left(g^{\prime} J\right)=\mathrm{h}_{S^{-1} \mathcal{O}_{\mathbb{K}}}\left(g^{\prime}\right)+\mathrm{h}_{S^{-1} \mathcal{O}_{\mathbb{K}}}(J)=\mathrm{h}_{S^{-1} \mathcal{O}_{\mathbb{K}}}(a)+\mathrm{h}_{S^{-1} \mathcal{O}_{\mathbb{K}}}(g J),
$$

where we again used the additivity of entropy. The right-hand side of (5) also satisfies this sort of additivity. We already know that (5) holds for the principal ideals $\left(g^{\prime}\right)$ and $(a)$. To conclude the proof we have to show it also holds for the primitive ideal $g J$.

Let $J$ be a primitive ideal. We define

$$
J^{\prime}=K J \cap L_{d}\left(S^{-1} \mathcal{O}_{\mathbb{K}}\right) \supseteq J,
$$

where the inclusion may be strict. We take a finite set $\left\{g_{1}, \ldots, g_{s}\right\}$ of generators for $J^{\prime}$. The ideal $J$ contains a primitive polynomial $f \in J$ as we have seen in Lemma 4.1. For $g_{i} \in K J$ there exists an $a_{i} \in S^{-1} \mathcal{O}_{\mathbb{K}}$ with $a_{i} g_{i} \in J$. With the ideals $J_{i}=\left\langle g_{1}, \ldots, g_{i}\right\rangle+J$ we get the exact sequences

$$
0 \rightarrow J_{i} / J \rightarrow J_{i+1} / J \rightarrow J_{i+1} / J_{i} \rightarrow 0 .
$$

The ideal $J_{i+1}$ is - modulo $J_{i}$ - generated by $g_{i+1}$. Therefore $J_{i+1} / J_{i}$ is isomorphic to the cyclic module 


$$
L_{d}\left(S^{-1} \mathcal{O}_{\mathbb{K}}\right) / I_{i}
$$

The ideal $I_{i}$ contains $f$ and $a_{i+1}$ because $f g_{i+1}, a_{i+1} g_{i+1} \in J \subseteq J_{i}$. From Lemma 4.4 we know that the entropy of the shift on the dual group of $J_{i+1} / J_{i}$ vanishes. Yuzvinskil's formula and induction show that the entropies of the shifts on the dual groups of $J_{i} / J$ vanish. In particular the entropy of the shift on $Y=\left(J^{\prime} / J\right)^{\wedge}$ is zero. The exact sequence

$$
0 \rightarrow J^{\prime} / J \rightarrow L_{d}\left(S^{-1} \mathcal{O}_{\mathbb{K}}\right) / J \rightarrow L_{d}\left(S^{-1} \mathcal{O}_{\mathbb{K}}\right) / J^{\prime} \rightarrow 0
$$

yields $\mathrm{h}_{S^{-1} \mathcal{O}_{\mathbb{K}}}(J)=\mathrm{h}_{S^{-1} \mathcal{O}_{\mathbb{K}}}\left(J^{\prime}\right)$.

Let $\left\{t_{1}, \ldots, t_{n}, \ldots\right\}$ be an enumeration of $S^{-1} \mathcal{O}_{\mathbb{K}} \backslash\{0\}$ and put

$$
r_{n}= \begin{cases}1 & \text { if } n=0, \\ \prod_{i=1}^{n} t_{i} & \text { if } n>0 .\end{cases}
$$

We define the abelian groups

$$
M_{n}=\frac{1}{r_{n}} S^{-1} \mathcal{O}_{\mathbb{K}}
$$

with union $\mathbb{K}$. The corresponding $L_{d}(\mathbb{Z})$-modules

$$
\sum_{\mathbb{Z}^{d}} M_{n} / K J \cap \sum_{\mathbb{Z}^{d}} M_{n}
$$

have $L_{d}(\mathbb{K}) / K J$ as union. Multiplication by $r_{n}$ shows they are all isomorphic to

$$
L_{d}\left(S^{-1} \mathcal{O}_{\mathbb{K}}\right) / J^{\prime}
$$

From Lemma 13.6 of [4] and Lemma 4.5 we get

$$
\mathrm{h}_{S^{-1} \mathcal{O}_{\mathbb{K}}}(J)=\mathrm{h}_{S^{-1} \mathcal{O}_{\mathbb{K}}}\left(J^{\prime}\right)=\mathrm{h}_{\mathbb{K}}(K J)=\mathrm{h}_{\mathbb{K}}\left(\operatorname{gcd}_{L_{d}(\mathbb{K})}(J)\right) .
$$

Therefore the problem reduces to the case of a principal ideal with $S^{\prime}=$ $\mathcal{O}_{\mathbb{K}} \backslash\{0\}$, and from the first part of the proof we get

$$
\mathrm{h}_{S^{-1} \mathcal{O}_{\mathbb{K}}}(J)=\mathrm{h}_{\mathbb{K}}\left(\operatorname{gcd}_{L_{d}(\mathbb{K})}(J)\right)=\log \left(\mathbb{M}\left(\operatorname{pr} N\left(\operatorname{gcd}_{L_{d}(\mathbb{K})}(J)\right)\right)\right) .
$$

The term $\mathcal{N}\left(\operatorname{Con}_{\mathbb{K}}(\cdot) \cap \mathcal{O}_{\mathbb{K}}\right)=1$ is trivial over the field $\mathbb{K}$. This is the formula we wanted to prove because for the primitive ideal $J$,

$$
\mathcal{N}\left(\operatorname{Con}_{S^{-1}} \mathcal{O}_{\mathbb{K}}(J) \cap \mathcal{O}_{\mathbb{K}}\right)=\mathcal{N}\left(S^{-1} \mathcal{O}_{\mathbb{K}} \cap \mathcal{O}_{\mathbb{K}}\right)=1 .
$$

4.7. EXAMPLE. For $\mathbb{K}=\mathbb{Q}(i)$ the domain of algebraic integers $\mathcal{O}_{\mathbb{K}}=\mathbb{Z}[i]$ is a principal ideal domain and we can compute the entropy by two different methods. We define $J=\left(f_{1}, f_{2}\right)$ to be the ideal with the generators

$$
f_{1}=(1+i)(2+i)\left(x^{2}-i\right), \quad f_{2}=(1-i)\left(x^{2}-2+i\right)\left(x^{2}-i\right) .
$$

The greatest common divisor of $f_{1}$ and $f_{2}$ is given by $(1+i)\left(x^{2}-i\right)$ which does not lie in $J$, because the two elements $2+i$ and $x^{2}-2+i$ do not generate the whole ring of polynomials. This can be seen by taking this ring 
modulo $(2+i)$, producing a ring isomorphic to $\mathbb{F}_{5}\left[x^{ \pm 1}\right]$. The second element $x^{2}-2+i$ now has the form $x^{2}-4$.

As $\mathbb{Z}[i]$ is a unique factorisation domain we can use Lemma 4.5 and Theorem 3.10 to compute the entropy

$$
\mathrm{h}_{\mathbb{Z}[i]}(J)=\log \mathbb{M}\left(2\left(x^{4}+1\right)\right)=\log 2 .
$$

But we can also use Theorem 4.6: the polynomials $f_{1}$ and $f_{2}$ have the greatest common divisor $x^{2}-i$ in the ring $\mathbb{Q}\left[i, x^{ \pm 1}\right]$ and the norm of this polynomial is $x^{4}+1$. As the ideal $\operatorname{Con}_{\mathbb{Z}[i]}(J)$ is generated by $\operatorname{Con}_{\mathbb{Z}[i]}\left(f_{1}\right)$ and $\mathrm{Con}_{\mathbb{Z}[i]}\left(f_{2}\right)$, it is equal to

$$
\operatorname{gcd}((1+i)(2+i),(1-i))=(1+i) .
$$

With the formula from Theorem 4.6 we get

$$
\mathrm{h}_{\mathbb{Z}[i]}(J)=\log \mathbb{M}\left(x^{4}+1\right)+\log \mathcal{N}(1+i)=\log 2 .
$$

Finally, we examine an example where Lemma 4.5 is not applicable.

ExAmple. Let $\mathbb{K}=\mathbb{Q}(\sqrt{-5})$. Then $\mathcal{O}_{\mathbb{K}}=\mathbb{Z}[\sqrt{-5}]$. We choose

$$
f_{1}=2\left(x^{2}-7\right), \quad f_{2}=(\sqrt{-5}+1)\left(x^{2}-7\right)\left(x^{2}-3\right)
$$

as the generators of $J=\left(f_{1}, f_{2}\right)$. Their greatest common divisor over $\mathbb{K}$ is $\left(x^{2}-7\right)$ and the content of $J$ is $(2, \sqrt{-5}+1)$. The entropy is given by

$$
\mathrm{h}_{\mathbb{Z}[\sqrt{-5}]}(J)=\log \mathbb{M}\left(N\left(x^{2}-7\right)\right)+\log \mathcal{N}(2, \sqrt{-5}+1)=\log 7+\log 2,
$$

where we have again used Theorem 4.6.

\section{References}

[1] D. Hilbert, Gesammelte Abhandlungen, Bd. I, Springer, Berlin, 1932.

[2] D. A. Lind, K. Schmidt and T. W ard, Mahler measure and entropy for commuting automorphisms of compact groups, Invent. Math. 101 (1990), 593-629.

[3] J. Neukirch, Algebraische Zahlentheorie, Springer, Berlin, 1992.

[4] K. Schmidt, Dynamical Systems of Algebraic Origin, Birkhäuser, 1995.

Mathematical Institute

University of Vienna

Strudlhofgasse 4

A-1090 Wien, Austria

E-mail: manfred@nelly.mat.univie.ac.at 